\title{
ASYMPTOTIC BEHAVIOR OF EIGENVALUES OF GREATEST COMMON DIVISOR MATRICES
}

\author{
SHAOFANG HONG \\ Mathematical College, Sichuan University, Chengdu 610064, P. R. China \\ e-mail: s-f.hong@163.net; hongsf02@yahoo.com \\ and RAPHAEL LOEWY \\ Department of Mathematics, Technion-Israel Institute of Technology, Haifa 32000, Israel \\ e-mail:loewy@techunix.technion.ac.il
}

(Received 3 October, 2003; accepted 22 January, 2004)

\begin{abstract}
Let $\left\{x_{i}\right\}_{i=1}^{\infty}$ be an arbitrary strictly increasing infinite sequence of positive integers. For an integer $n \geq 1$, let $S_{n}=\left\{x_{1}, \ldots, x_{n}\right\}$. Let $\varepsilon$ be a real number and $q \geq 1$ a given integer. Let $\lambda_{n}^{(1)} \leq \cdots \leq \lambda_{n}^{(n)}$ be the eigenvalues of the power GCD matrix $\left(\left(x_{i}, x_{j}\right)^{\varepsilon}\right)$ having the power $\left(x_{i}, x_{j}\right)^{\varepsilon}$ of the greatest common divisor of $x_{i}$ and $x_{j}$ as its $i, j$-entry. We give a nontrivial lower bound depending on $x_{1}$ and $n$ for $\lambda_{n}^{(1)}$ if $\varepsilon>0$. Especially for $\varepsilon>1$, this lower bound is given by using the Riemann zeta function. Let $x \geq 1$ be an integer. For a sequence $\left\{x_{i}\right\}_{i=1}^{\infty}$ satisfying that $\left(x_{i}, x_{j}\right)=x$ for any $i \neq j$ and $\sum_{i=1}^{\infty} \frac{1}{x_{i}}=\infty$, we show that if $0<\varepsilon \leq 1$, then $\lim _{n \rightarrow \infty} \lambda_{n}^{(1)}=x_{1}^{\varepsilon}-x^{\varepsilon}$. Let $a \geq 0, b \geq 1$ and $e \geq 0$ be any given integers. For the arithmetic progression $\left\{x_{i-e+1}=a+b i\right\}_{i=e}^{\infty}$, we show that if $0<\varepsilon \leq 1$, then $\lim _{n \rightarrow \infty} \lambda_{n}^{(q)}=0$. Finally, we show that for any sequence $\left\{x_{i}\right\}_{i=1}^{\infty}$ and any $\varepsilon>0, \lambda_{n}^{(n-q+1)}$ approaches infinity when $n$ goes to infinity.
\end{abstract}

2000 Mathematics Subject Classification. 11C20, 11A05, 15A36.

1. Introduction. In $1876, \mathrm{H}$. Smith [25] published his celebrated theorem showing that the determinant of the $n \times n$ matrix $[(i, j)]$, which has the greatest common divisor $(i, j)$ of $i$ and $j$ as its $(i, j)$-entry, is the product $\prod_{k=1}^{n} \varphi(k)$, where $\varphi$ is Euler's totient function. Smith also proved that if $f$ is an arithmetical function and $[f(i, j)]$ is the $n \times n$ matrix having $f$ evaluated at the greatest common divisor $(i, j)$ of $i$ and $j$ as its $(i, j)$-entry, then $\operatorname{det}[f(i, j)]=\prod_{k=1}^{n}(f * \mu)(k)$, where $\mu$ is the Möbius function and $f * \mu$ is the Dirichlet convolution of $f$ and $\mu$. In 1972, Apostol [2] extended Smith's result. In 1988, McCarthy [21] generalized Smith's and Apostol's results to the class of even functions of $m(\bmod r)$, where $m$ and $r$ are positive integers. A complex-valued function $\beta(m, r)$ is said to be an even function of $m(\bmod r)$ if $\beta(m, r)=\beta((m, r), r)$ for all values of $m$. The functions considered by Smith and Apostol are even functions of $m(\bmod r)$. In 1993, Bourque and Ligh [5] extended the results of Smith, Apostol, and McCarthy. In 2002, Hong [12] generalized the results of Smith, Apostol, McCarthy and Bourque and Ligh to certain classes of arithmetical functions. In 2003, Korkee and Haukkanen [18] considered a certain abstract generalization of Smith's determinant. 
Let $1 \leq x_{1}<\cdots<x_{n}<\cdots$ be a given arbitrary strictly increasing infinite sequence of positive integers. For any integer $n \geq 1$, let

$$
S_{n}=\left\{x_{1}, \ldots, x_{n}\right\}
$$

Denote by $\left(f\left(x_{i}, x_{j}\right)\right)$ the $n \times n$ matrix having $f$ evaluated at the greatest common divisor $\left(x_{i}, x_{j}\right)$ of $x_{i}$ and $x_{j}$ as its $i, j$-entry and by $\left(f\left[x_{i}, x_{j}\right]\right)$ denote the $n \times n$ matrix having $f$ evaluated at the least common multiple $\left[x_{i}, x_{j}\right]$ of $x_{i}$ and $x_{j}$ as its $i, j$-entry. The set $S_{n}$ is said to be factor closed if it contains every divisor of $x$ for any $x \in S_{n}$. From Bourque and Ligh's result [6, Theorem 4], we can see that if $S_{n}$ is a factor-closed set and $f$ is a multiplicative function such that $f \in \mathcal{L}_{S_{n}}$, where $\mathcal{L}_{S_{n}}$ is a certain class of arithmetical functions defined by

$$
\mathcal{L}_{S_{n}}:=\left\{f:(f * \mu)(d) \in \mathbf{Z} \backslash\{0\} \text { whenever } d \mid \operatorname{lcm}\left(S_{n}\right)\right\},
$$

where $\operatorname{lcm}\left(S_{n}\right)$ means the least common multiple of all elements in $S_{n}$, then the matrix $\left(f\left(x_{i}, x_{j}\right)\right)$ divides the matrix $\left(f\left[x_{i}, x_{j}\right]\right)$ in the ring $M_{n}(\mathbf{Z})$ of $n \times n$ matrices over the integers. Hong [13] showed that for any multiple-closed set $S_{n}$ (i.e. $y \in S_{n}$ whenever $x|y| \operatorname{lcm}\left(S_{n}\right)$ for any $\left.x \in S_{n}\right)$ and for any divisor chain $S_{n}$ (i.e. $\left.x_{1}|\cdots| x_{n}\right)$, if $f$ is a completely multiplicative function such that $f \in \mathcal{L}_{S_{n}}$, then the matrix $\left(f\left(x_{i}, x_{j}\right)\right)$ divides the matrix $\left(f\left[x_{i}, x_{j}\right]\right)$ in the ring $M_{n}(\mathbf{Z})$. But such a factorization is no longer true if $f$ is multiplicative.

Now let $\varepsilon$ be a real number. The $n \times n$ matrix having the power $\left(x_{i}, x_{j}\right)^{\varepsilon}$ of the greatest common divisor of $x_{i}$ and $x_{j}$ as its $i, j$-entry is called the power greatest common divisor $(G C D)$ matrix defined on $S_{n}$, denoted by $\left(\left(x_{i}, x_{j}\right)^{\varepsilon}\right)$, or abbreviated by $\left(\left(S_{n}\right)^{\varepsilon}\right)$. The matrix having the power $\left[x_{i}, x_{j}\right]^{\varepsilon}$ of the least common multiple of $x_{i}$ and $x_{j}$ as its $i, j$ entry is called the power least common multiple $(L C M)$ matrix, denoted by $\left(\left[x_{i}, x_{j}\right]^{\varepsilon}\right)$, or abbreviated by $\left[\left(S_{n}\right)^{\varepsilon}\right]$. If we let $\varepsilon=1$, then the power GCD matrix and the power LCM matrix are said to be the GCD matrix defined on $S_{n}$ and the LCM matrix defined on $S_{n}$, respectively, and denoted by $\left(S_{n}\right)$ and [ $\left.S_{n}\right]$, respectively. In 1989, Beslin and Ligh [3] initiated the study of the GCD matrix $\left(S_{n}\right)$ on any set $S_{n}$ in the direction of structure, determinant and inverse. In particular, they proved that the GCD matrix $\left(S_{n}\right)$ on any set $S_{n}$ of $n$ distinct positive integers is positive definite. However, the LCM matrix $\left[S_{n}\right]$ on any set $S_{n}$ is not positive definite in general. It may even not be nonsingular. In fact, Hong [11] showed that for any integer $n \geq 8$, there exists a GCD-closed set $S_{n}=\left\{x_{1}, \ldots, x_{n}\right\}$ (i.e. one has $\left(x_{i}, x_{j}\right) \in S_{n}$ for all $\left.1 \leq i, j \leq n\right)$ such that the LCM matrix $\left[S_{n}\right]$ on $S_{n}$ is singular. Note also that recently, Hong [14] proved that for any positive integer $\varepsilon$ and for any GCD-closed set $S_{n}$ satisfying $\max _{x \in S_{n}}\{v(x)\} \leq 2$, where $v(x)$ denotes the number of distinct prime factors of the positive integer $x$, the power LCM matrix $\left[\left(S_{n}\right)^{\varepsilon}\right]$ on $S_{n}$ is nonsingular.

For a different form of a power GCD matrix

$$
N_{n}:=\left(\frac{(i, j)^{2 \varepsilon}}{i^{\varepsilon} \cdot j^{\varepsilon}}\right)_{1 \leq i, j \leq n},
$$

Wintner [26] proved in 1944 that $\lim _{\sup _{n \rightarrow \infty}} \Lambda_{n}(\varepsilon)<\infty$ if and only if $\varepsilon>1$, where $\Lambda_{n}(\varepsilon)$ denotes the largest eigenvalue of the matrix $N_{n}$. Let $\lambda_{n}(\varepsilon)$ denote the smallest eigenvalue of the matrix $N_{n}$. Lindqvist and Seip [19] in 1998 use the work of [9] about Riesz bases to investigate the asymptotic behavior of $\lambda_{n}(\varepsilon)$ and $\Lambda_{n}(\varepsilon)$ as $n \rightarrow \infty$. In particular, they got a sharp bound for $\lambda_{n}(\varepsilon)$ and $\Lambda_{n}(\varepsilon)$. However, for the power GCD 
matrix $\left(\left(S_{n}\right)^{\varepsilon}\right)$ on $S_{n}$, the eigenvalues do not seem to be known. In 1993, Bourque and Ligh [5] extended Beslin and Ligh's result by showing that for any $\varepsilon>0$, the power GCD matrix $\left(\left(S_{n}\right)^{\varepsilon}\right)$ on $S_{n}$ is positive definite. From this, one can only conclude that its eigenvalues are positive, but no further information is provided.

In the present paper, our main goal is to consider the asymptotic behavior of the eigenvalues of the power GCD matrix $\left(\left(S_{n}\right)^{\varepsilon}\right)$ on $S_{n}$. Let $\lambda_{n}^{(1)} \leq \cdots \leq \lambda_{n}^{(n)}$ be the eigenvalues of the power GCD matrix $\left(\left(x_{i}, x_{j}\right)^{\varepsilon}\right)$ defined on the set $\left\{x_{1}, \ldots, x_{n}\right\}$. Let $1 \leq q \leq n$ be a fixed integer and $\varepsilon>0$. Then it follows from Bourque and Ligh's result [4] that

$$
\lambda_{n}^{(q)}>0
$$

On the other hand, by Cauchy's interlacing inequalities (see [15]) we have

$$
\lambda_{n+1}^{(q)} \leq \lambda_{n}^{(q)}
$$

Thus the sequence $\left\{\lambda_{n}^{(q)}\right\}_{n=q}^{\infty}$ is a non-increasing infinite sequence of positive real numbers and so it is convergent. Namely, we have the following result.

Proposition 1.1. Let $q \geq 1$ be a given arbitrary integer, $\varepsilon>0$ and $\left\{x_{i}\right\}_{i=1}^{\infty}$ an arbitrary given strictly increasing infinite sequence of positive integers. Let $\lambda_{n}^{(1)} \leq \cdots \leq \lambda_{n}^{(n)}$ be the eigenvalues of the $n \times n$ power GCD matrix $\left(\left(x_{i}, x_{j}\right)^{\varepsilon}\right)$ defined on the set $\left\{x_{1}, \ldots, x_{n}\right\}$. Then the sequence $\left\{\lambda_{n}^{(q)}\right\}_{n=q}^{\infty}$ converges and

$$
\lim _{n \rightarrow \infty} \lambda_{n}^{(q)} \geq 0
$$

Let $x \geq 1$ be an integer. For an arbitrary strictly increasing infinite sequence $\left\{x_{i}\right\}_{i=1}^{\infty}$ of positive integers satisfying that $\left(x_{i}, x_{j}\right)=x$ for any $i \neq j$ and $\sum_{i=1}^{\infty} \frac{1}{x_{i}}=\infty$, we show, in section 2 , that if $0<\varepsilon \leq 1$, then $\lim _{n \rightarrow \infty} \lambda_{n}^{(1)}=x_{1}^{\varepsilon}-x^{\varepsilon}$. Let $a \geq 0, b \geq 1$ and $e \geq 0$ be any given integers. In section 3 , we show that for the arithmetic progression $\left\{x_{i-e+1}=a+b i\right\}_{i=e}^{\infty}$, if $0<\varepsilon \leq 1$, then $\lim _{n \rightarrow \infty} \lambda_{n}^{(q)}=0$.

We give in Section 4 a lower bound for the smallest eigenvalue $\lambda_{n}^{(1)}$ of the power GCD matrix $\left(\left(S_{n}\right)^{\varepsilon}\right)$ on any set $S_{n}$. This improves the lower bound due to Beslin, Bourque and Ligh. Then we use it to obtain a lower bound for the $q$-th largest eigenvalue $\lambda_{n}^{(n-q+1)}$ of the power GCD matrix $\left(\left(S_{n}\right)^{\varepsilon}\right)$ on any set $S_{n}$ for any $\varepsilon>0$ and any given integer $q \geq 1$. This lower bound then implies that for any $\varepsilon>0$ and any given integer $q \geq 1$, the $q$-th largest eigenvalue of the power GCD matrix $\left(\left(S_{n}\right)^{\varepsilon}\right)$ on any set $S_{n}$ tends to infinity as $n$ tends to infinity. The final section contains some remarks and questions.

For a comprehensive review of papers relating to greatest common divisor matrices not presented here, we refer the readers to [8]. Throughout this paper, we let $E_{n}$ denote the $n \times n$ matrix with all entries equal to 1 .

2. Some preliminary results. In this section we shall study the asymptotic behavior of the smallest eigenvalue of the power GCD matrix defined on the ordered finite subsequence of an infinite sequence of pairwise relatively prime positive integers. First we state some results on certain symmetric matrices. The following lemma is known. 
LEMMA 2.1. Let $n \geq 1$ be an integer and let $a_{1}, \ldots, a_{n} \in \mathbf{F}$ such that $a_{i} \neq 1$ for all $1 \leq i \leq n$, where $\mathbf{F}$ is an arbitrary field. Then

$$
\left(\begin{array}{cccc}
1 & 1 & \cdots & 1 \\
1 & a_{1} & \cdots & 1 \\
. & . . & . . & . . \\
1 & 1 & \cdots & a_{n}
\end{array}\right)^{-1}=\left(\begin{array}{ccccc}
1+\sum_{i=1}^{n} \frac{1}{a_{i}-1} & -\frac{1}{a_{1}-1} & -\frac{1}{a_{2}-1} & \cdots & -\frac{1}{a_{n}-1} \\
-\frac{1}{a_{1}-1} & \frac{1}{a_{1}-1} & 0 & \cdots & 0 \\
-\frac{1}{a_{2}-1} & 0 & \frac{1}{a_{2}-1} & \cdots & 0 \\
\cdots & \cdots & \cdots & \cdots & \cdots \\
-\frac{1}{a_{n}-1} & 0 & 0 & \cdots & \frac{1}{a_{n}-1}
\end{array}\right) .
$$

Assume now that $\left\{u_{i}\right\}_{i=1}^{\infty}$ is any given strictly increasing infinite sequence of real numbers such that $u_{1}>1$. Let $U_{n}:=E_{n}+\operatorname{diag}\left(0, u_{1}-1, \ldots, u_{n-1}-1\right)$. It is easy to see that the $n \times n$ matrix $U_{n}$ is positive definite. Let $\lambda_{n}^{(1)} \leq \cdots \leq \lambda_{n}^{(n)}$ be the eigenvalues of the $n \times n$ matrix $U_{n}$. Then $\lambda_{n}^{(1)}>0$. Now let $\mu_{n}^{(1)} \leq \cdots \leq \mu_{n}^{(n)}$ be the eigenvalues of the inverse matrix $U_{n}^{-1}$. Then

$$
\lambda_{n}^{(i)} \cdot \mu_{n}^{(n-i+1)}=1, \quad 1 \leq i \leq n
$$

LEMMA 2.2. Suppose that $\left\{u_{i}\right\}_{i=1}^{\infty}$ is any given strictly increasing infinite sequence of real numbers such that $u_{1}>1$. Let $U_{n}=E_{n}+\operatorname{diag}\left(0, u_{1}-1, \ldots, u_{n-1}-1\right)$ and $\mu_{n}^{(n)}$ the largest eigenvalue of the inverse matrix $U_{n}^{-1}$. Then

$$
\mu_{n}^{(n)}>1+\sum_{i=1}^{n-1} \frac{1}{u_{i}-1}
$$

Proof. Define an $n \times n$ matrix $V_{n}$ as follows:

$$
V_{n}=\left(\begin{array}{cccc}
1+\sum_{i=1}^{n} \frac{1}{u_{i}-1} & -\frac{1}{u_{1}-1} & \cdots & -\frac{1}{u_{n-1}-1} \\
-\frac{1}{u_{1}-1} & 0 & \cdots & 0 \\
\cdots & \cdots & \cdots & \cdots \\
-\frac{1}{u_{n-1}-1} & 0 & \cdots & 0
\end{array}\right) .
$$

Then the characteristic polynomial of $V_{n}$ is given by

$$
\left|\lambda I_{n}-V_{n}\right|=\lambda^{n-2}\left(\lambda^{2}-\left(1+\sum_{i=1}^{n-1} \frac{1}{u_{i}-1}\right) \lambda-\sum_{i=1}^{n-1} \frac{1}{\left(u_{i}-1\right)^{2}}\right) .
$$

So the largest eigenvalue $\lambda_{\max }\left(V_{n}\right)$ of $V_{n}$ satisfies

$$
\lambda_{\max }\left(V_{n}\right)>1+\sum_{i=1}^{n-1} \frac{1}{u_{i}-1} .
$$


By Lemma 2.1 one gets

$$
U_{n}^{-1}=\left(\begin{array}{ccccc}
1+\sum_{i=1}^{n} \frac{1}{u_{i}-1} & -\frac{1}{u_{1}-1} & -\frac{1}{u_{2}-1} & \cdots & -\frac{1}{u_{n-1}-1} \\
-\frac{1}{u_{1}-1} & \frac{1}{u_{1}-1} & 0 & \cdots & 0 \\
-\frac{1}{u_{2}-1} & 0 & \frac{1}{u_{2}-1} & \cdots & 0 \\
\cdots & \ldots & \cdots & \cdots & \cdots \\
-\frac{1}{u_{n-1}-1} & 0 & 0 & \cdots & \frac{1}{u_{n-1}-1}
\end{array}\right) .
$$

Replacing all negative terms $-\frac{1}{u_{i}-1}(1 \leq i \leq n)$ by $\frac{1}{u_{i}-1}$ in the matrices $V_{n}$ and $U_{n}^{-1}$, we get the corresponding nonnegative (element-wise) matrices $\left|V_{n}\right|$ and $\left|U_{n}^{-1}\right|$, respectively. Clearly $V_{n},\left|V_{n}\right|$ are similar, and $U_{n}^{-1},\left|U_{n}^{-1}\right|$ likewise. Thus the spectral radii satisfy

$$
\rho\left(\left|V_{n}\right|\right)=\rho\left(V_{n}\right)=\lambda_{\max }\left(V_{n}\right)
$$

and

$$
\rho\left(\left|U_{n}^{-1}\right|\right)=\rho\left(U_{n}^{-1}\right)=\mu_{n}^{(n)} .
$$

Since $0 \leq\left|V_{n}\right| \leq\left|U_{n}^{-1}\right|$, one deduces immediately from the Perron-Frobenius theorem for nonnegative matrices (see, for example, [15]) that $\rho\left(\left|V_{n}\right|\right) \leq \rho\left(U_{n}^{-1}\right)$. So we have

$$
\lambda_{\max }\left(V_{n}\right) \leq \mu_{n}^{(n)} .
$$

The result follows from (2.2) and (2.3).

COROLlary 2.3. Suppose that $\left\{u_{i}\right\}_{i=1}^{\infty}$ is any given strictly increasing infinite sequence of real numbers such that $u_{1}>1$ and

$$
\sum_{i=1}^{\infty} \frac{1}{u_{i}}=\infty
$$

Let $\lambda_{n}^{(1)}$ be the smallest eigenvalue of the $n \times n$ matrix $U_{n}=E_{n}+\operatorname{diag}\left(0, u_{1}-\right.$ $\left.1, \ldots, u_{n-1}-1\right)$. Then

$$
\lim _{n \rightarrow \infty} \lambda_{n}^{(1)}=0
$$

Proof. Let $\mu_{n}^{(n)}$ be the largest eigenvalue of the inverse matrix $U_{n}^{-1}$. By (2.1)

$$
\lambda_{n}^{(1)}=\frac{1}{\mu_{n}^{(n)}} .
$$

It then follows from Lemma 2.2 that for $n \geq 2$,

$$
\lambda_{n}^{(1)}<\frac{1}{1+\sum_{i=1}^{n-1} \frac{1}{u_{i}-1}}<\frac{1}{1+\sum_{i=1}^{n-1} \frac{1}{u_{i}}} .
$$

Since $\lambda_{n}^{(1)}>0$ it follows from (2.4) and (2.5) that $\lim _{n \rightarrow \infty} \lambda_{n}^{(1)}=0$. 
COROLLARY 2.4. Let $\left\{r_{i}\right\}_{i=1}^{\infty}$ be a strictly increasing infinite sequence of real numbers satisfying $r_{1} \geq 1$ and

$$
\sum_{i=1}^{\infty} \frac{1}{r_{i}}=\infty
$$

Let $\lambda_{n}^{(1)}$ denote the smallest eigenvalue of the $n \times n$ matrix $E_{n}+\operatorname{diag}\left(r_{1}-1, \ldots, r_{n}-1\right)$. Then

$$
\lim _{n \rightarrow \infty} \lambda_{n}^{(1)}=r_{1}-1
$$

Proof. Let

$$
R_{n}:=E_{n}+\operatorname{diag}\left(r_{1}-1, \ldots, r_{n}-1\right)
$$

Then

$$
R_{n}=\left(r_{1}-1\right) I_{n}+E_{n}+\operatorname{diag}\left(0,\left(r_{2}-r_{1}+1\right)-1, \ldots,\left(r_{n}-r_{1}+1\right)-1\right) .
$$

Since $r_{2}-r_{1}+1>1$ and

$$
\sum_{i=2}^{\infty} \frac{1}{r_{i}-r_{1}+1}=\infty
$$

the result follows immediately from Corollary 2.3.

The following is the main result of this section.

THEOREM 2.5. Let $0<\varepsilon \leq 1$ and $x$ a positive integer. Let $\left\{x_{i}\right\}_{i=1}^{\infty}$ be a strictly increasing infinite sequence of positive integers satisfying the following conditions.

(i) For every $i \neq j,\left(x_{i}, x_{j}\right)=x$;

(ii) $\sum_{i=1}^{\infty} \frac{1}{x_{i}}=\infty$.

If $\lambda_{n}^{(1)}$ is the smallest eigenvalue of the $n \times n$ power $G C D$ matrix $\left(\left(x_{i}, x_{j}\right)^{\varepsilon}\right)$ defined on the set $\left\{x_{1}, \ldots, x_{n}\right\}$, then

$$
\lim _{n \rightarrow \infty} \lambda_{n}^{(1)}=x_{1}^{\varepsilon}-x^{\varepsilon}
$$

Proof. For $i \geq 1$, let $x_{i}=x \cdot \bar{x}_{i}$. Note that $x>0$. Then from (i) and (ii) we can easily deduce that for any $i \neq j,\left(\bar{x}_{i}, \bar{x}_{j}\right)=1$ and

$$
\sum_{i=1}^{\infty} \frac{1}{\bar{x}_{i}}=\infty
$$

Obviously we have

$$
\left(\left(\bar{x}_{i}, \bar{x}_{j}\right)^{\varepsilon}\right)=E_{n}+\operatorname{diag}\left(\bar{x}_{1}^{\varepsilon}-1, \ldots, \bar{x}_{n}^{\varepsilon}-1\right) .
$$


For $1 \leq i \leq n$, let $r_{i}=\bar{x}_{i}^{\varepsilon}$. Then $r_{1}=\bar{x}_{1}^{\varepsilon} \geq 1$ and for every $1 \leq i \leq n, r_{i} \leq \bar{x}_{i}$. Hence we have by (2.6)

$$
\sum_{i=1}^{\infty} \frac{1}{r_{i}}=\infty
$$

Let $\bar{\lambda}_{n}^{(1)}$ be the smallest eigenvalue of the $n \times n$ power GCD matrix $\left(\left(\bar{x}_{i}, \bar{x}_{j}\right)^{\varepsilon}\right)$ defined on the set $\left\{\bar{x}_{1}, \ldots, \bar{x}_{n}\right\}$. Thus by Corollary 2.4 we have $\lim _{n \rightarrow \infty} \bar{\lambda}_{n}^{(1)}=\bar{x}_{1}^{\varepsilon}-1$. The result then follows immediately from the fact that $\lambda_{n}^{(1)}=x^{\varepsilon} \cdot \bar{\lambda}_{n}^{(1)}$.

3. Arithmetic progressions and the $q$-th smallest eigenvalue. In this section, we turn our attention to arithmetic progressions. First we introduce the following concept.

Definition. Let $e$ and $r$ be positive integers. Let $X=\left\{x_{1}, \ldots, x_{e}\right\}$ and $Y=$ $\left\{y_{1}, \ldots, y_{r}\right\}$ be two sets of distinct positive integers. Then we define the tensor product (set) of $X$ and $Y$, denoted by $X \odot Y$, by

$$
X \odot Y:=\left\{x_{1} y_{1}, \ldots, x_{1} y_{r}, x_{2} y_{1}, \ldots, x_{2} y_{r}, \ldots, x_{e} y_{1}, \ldots, x_{e} y_{r}\right\} .
$$

REMARK. It must be pointed out that the elements in the tensor product set are not necessarily arranged in increasing order. For example, let $X=\{1,2,3\}$ and $Y=$ $\{3,5\}$. Then $X \odot Y=\{3,5,6,10,9,15\}$. We note also that the elements in the tensor product set are not necessarily distinct. For example, let $X=\{2,3\}$ and $Y=\{4,6\}$. Then $X \odot Y=\{8,12,12,18\}$.

LEMMA 3.1. Let $\varepsilon$ be any real number. Let $e$ and $r$ be positive integers. Let $X=\left\{x_{1}, \ldots, x_{e}\right\}$ be a set of e distinct positive integers such that for any $1 \leq i \neq j \leq e$, $\left(x_{i}, x_{j}\right)=1$. Let $Y=\left\{y_{1}, \ldots, y_{r}\right\}$ be a set of $r$ distinct positive integers such that for any $1 \leq i \neq j \leq r,\left(y_{i}, y_{j}\right)=1$. Assume that for all $1 \leq i \leq e, 1 \leq j \leq r,\left(x_{i}, y_{j}\right)=1$. Then the following equality holds:

$$
\left((X \odot Y)^{\varepsilon}\right)=\left((X)^{\varepsilon}\right) \otimes\left((Y)^{\varepsilon}\right)
$$

Proof. First we have

$$
\left((X)^{\varepsilon}\right)=\left(\begin{array}{cccc}
x_{1}^{\varepsilon} & 1 & \cdots & 1 \\
1 & x_{2}^{\varepsilon} & \cdots & 1 \\
. . & . . & . . & . . \\
1 & 1 & \cdots & x_{e}^{\varepsilon}
\end{array}\right) \quad \text { and }\left((Y)^{\varepsilon}\right)=\left(\begin{array}{cccc}
y_{1}^{\varepsilon} & 1 & \cdots & 1 \\
1 & y_{2}^{\varepsilon} & \cdots & 1 \\
. \cdot & . . & . . & . . \\
1 & 1 & \cdots & y_{r}^{\varepsilon}
\end{array}\right) .
$$

Since

$$
\left(x_{i_{1}} y_{j_{1}}, x_{i_{2}} y_{j_{2}}\right)=\left\{\begin{array}{cl}
x_{i_{1}} y_{j_{1}} & \text { if } i_{1}=i_{2} \text { and } j_{1}=j_{2} \\
y_{j_{1}} & \text { if } i_{1} \neq i_{2} \text { and } j_{1}=j_{2} \\
x_{i_{1}} & \text { if } i_{1}=i_{2} \text { and } j_{1} \neq j_{2} \\
1 & \text { if } i_{1} \neq i_{2} \text { and } j_{1} \neq j_{2}
\end{array}\right.
$$


letting $Y_{r}=\left((Y)^{\varepsilon}\right)$ we get

$$
\begin{aligned}
\left((X \odot Y)^{\varepsilon}\right) & =\left(\begin{array}{cccc}
x_{1}^{\varepsilon} Y_{r} & Y_{r} & \cdots & Y_{r} \\
Y_{r} & x_{2}^{\varepsilon} Y_{r} & \cdots & Y_{r} \\
. . & . . & . . & . . \\
Y_{r} & Y_{r} & \cdots & x_{e}^{\varepsilon} Y_{r}
\end{array}\right) \\
& =\left(\begin{array}{cccc}
x_{1}^{\varepsilon} & 1 & \cdots & 1 \\
1 & x_{2}^{\varepsilon} & \cdots & 1 \\
. . & . . & . . & . . \\
1 & 1 & \cdots & x_{e}^{\varepsilon}
\end{array}\right) \otimes Y_{r} \\
& =\left((X)^{\varepsilon}\right) \otimes\left((Y)^{\varepsilon}\right)
\end{aligned}
$$

as desired.

LEMMA 3.2. Let $b \geq 1$ be an integer and $0<\varepsilon \leq 1$. Let $\lambda_{n}^{(1)} \leq \cdots \leq \lambda_{n}^{(n)}$ be the eigenvalues of the $n \times n$ power $G C D$ matrix $\left((1+b i, 1+b j)^{\varepsilon}\right)$ defined on the set $\{1+b i\}_{i=0}^{n-1}$. Then for any given integer $q \geq 1$ we have

$$
\lim _{n \rightarrow \infty} \lambda_{n}^{(q)}=0 .
$$

Proof. By Dirichlet's theorem (see [1], or [17]) there are infinitely many primes in the arithmetic progression $\{1+b i\}_{i=0}^{\infty}$. Let $p_{1}<\cdots<p_{n}<\cdots$ denote the primes in this arithmetic progression. By Mertens' theorem (see [22])

$$
\sum_{i=1}^{\infty} \frac{1}{p_{i}}=\infty,
$$

and since $0<\varepsilon \leq 1$, it follows that

$$
\sum_{i=1}^{\infty} \frac{1}{p_{i}^{\varepsilon}}=\infty .
$$

For $i \geq 1$, let $\pi_{i}=p_{q-1+i}$. Then $p_{q-1}<\pi_{1}<\pi_{2}<\cdots$. Since $q$ is a fixed number, it follows from (3.1) that

$$
\sum_{i=1}^{\infty} \frac{1}{\pi_{i}^{\varepsilon}}=\infty
$$

Now let $r \geq 2$ be an arbitrary integer and let

$$
P_{q}:=\left\{1, p_{1}, \ldots, p_{q-1}\right\}, \quad T_{r}:=\left\{1, \pi_{1}, \ldots, \pi_{r-1}\right\} .
$$

Consider the tensor product set $P_{q} \odot T_{r}$. Note that the entries in the set $P_{q} \odot T_{r}$ are not arranged in increasing order, but the eigenvalues of the corresponding power GCD matrix do not depend on rearranging those entries. By Lemma 3.1

$$
\left(\left(P_{q} \odot T_{r}\right)^{\varepsilon}\right)=\left(\left(P_{q}\right)^{\varepsilon}\right) \otimes\left(\left(T_{r}\right)^{\varepsilon}\right) .
$$


Let $\mu_{q}^{(1)} \leq \cdots \leq \mu_{q}^{(q)}$ and $\tilde{\lambda}_{r}^{(1)} \leq \cdots \leq \tilde{\lambda}_{r}^{(r)}$ be the eigenvalues of the power GCD matrix $\left(\left(P_{q}\right)^{\varepsilon}\right)$ defined on the set $P_{q}$ and the power GCD matrix $\left(\left(T_{r}\right)^{\varepsilon}\right)$ defined on the set $T_{r}$ respectively. Then it is known (see [16]) that the eigenvalues of the tensor product matrix $\left(\left(P_{q}\right)^{\varepsilon}\right) \otimes\left(\left(T_{r}\right)^{\varepsilon}\right)$ are given by the set

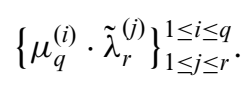

Notice that

$$
\mu_{q}^{(1)} \cdot \tilde{\lambda}_{r}^{(1)} \leq \cdots \leq \mu_{q}^{(q)} \cdot \tilde{\lambda}_{r}^{(1)} .
$$

Since for any $i_{1}, i_{2} \in \mathbf{Z}$,

$$
\left(1+b i_{1}\right)\left(1+b i_{2}\right)=1+b i_{1}+b i_{2}+b^{2} i_{1} i_{2} \equiv 1 \quad(\bmod b),
$$

the arithmetic progression $\{1+b i\}_{i=0}^{\infty}$ is closed under the usual multiplication. So the tensor product set $P_{q} \odot T_{r} \subset\{1+b i\}_{i=0}^{\infty}$. For any integer $r \geq 2$, define an integer $n_{r}$ by

$$
n_{r}:=\frac{p_{q-1} \cdot \pi_{r-1}-1}{b}+1 .
$$

Then $P_{q} \odot T_{r} \subseteq\{1+b i\}_{i=0}^{n_{r}-1}$. Thus the power GCD matrix $\left(\left(P_{q} \odot T_{r}\right)^{\varepsilon}\right)$ defined on $P_{q} \odot T_{r}$ is a principal submatrix of the $n_{r} \times n_{r}$ power GCD matrix $\left((1+b i, 1+b j)^{\varepsilon}\right)$ defined on the set $\left\{1,1+b, \ldots, 1+b\left(n_{r}-1\right)\right\}$. Let $\bar{\lambda}_{q r}^{(1)} \leq \cdots \leq \bar{\lambda}_{q r}^{(q r)}$ be the eigenvalues of $\left(\left(P_{q} \odot T_{r}\right)^{\varepsilon}\right)$. Then by Cauchy's interlacing inequalities we have

$$
\lambda_{n_{r}}^{(q)} \leq \bar{\lambda}_{q r}^{(q)} .
$$

But by (3.3)

$$
\bar{\lambda}_{q r}^{(q)} \leq \mu_{q}^{(q)} \cdot \tilde{\lambda}_{r}^{(1)} .
$$

So it follows from (3.4) and (3.5) that

$$
\lambda_{n_{r}}^{(q)} \leq \mu_{q}^{(q)} \cdot \tilde{\lambda}_{r}^{(1)} .
$$

On the other hand, in Theorem 2.5, if we choose $x=x_{1}=1$ and $x_{i}=\pi_{i-1}$ for $i \geq 2$, then by (3.2) the two conditions of Theorem 2.5 are satisfied. It then follows immediately from Theorem 2.5 that

$$
\lim _{r \rightarrow \infty} \tilde{\lambda}_{r}^{(1)}=0 .
$$

It follows from Proposition 1.1 that the subsequence $\left\{\lambda_{n_{r}}^{(q)}\right\}_{r=1}^{\infty}$ of the sequence $\left\{\lambda_{n}^{(q)}\right\}_{n=1}^{\infty}$ converges and

$$
\lim _{r \rightarrow \infty} \lambda_{n_{r}}^{(q)} \geq 0
$$

Hence by (3.6)-(3.8), $\lim _{r \rightarrow \infty} \lambda_{n_{r}}^{(q)}=0$. Finally, again by Proposition 1.1, the desired result $\lim _{n \rightarrow \infty} \lambda_{n}^{(q)}=0$ follows immediately.

We are now in a position to give the main result of this section. 
THEOREM 3.3. Let $a, b \geq 1$ and $e \geq 0$ be integers and $0<\varepsilon \leq 1$. Let $\lambda_{n}^{(1)} \leq \cdots \leq \lambda_{n}^{(n)}$ be the eigenvalues of the $n \times n$ power GCD matrix $\left((a+b i, a+b j)^{\varepsilon}\right)$ defined on the set $\{a+b e, a+b(e+1), \ldots, a+b(e+n-1)\}$. Then for any given integer $q \geq 1$

$$
\lim _{n \rightarrow \infty} \lambda_{n}^{(q)}=0
$$

Proof. For the arithmetic progression $\{a+b i\}_{i=e}^{\infty}$, consider its subsequence

$$
\{a+b(e+(a+b e) i)\}_{i=0}^{\infty}=\{(a+b e)(1+b i)\}_{i=0}^{\infty} .
$$

For any integer $m \geq 1$, let $\mu_{m}^{(1)} \leq \cdots \leq \mu_{m}^{(m)}$ be the eigenvalues of the $m \times m$ power GCD matrix $\left(\left(W_{m}\right)^{\varepsilon}\right)$ defined on the set

$$
W_{m}:=\{a+b e,(a+b e)(1+b), \ldots,(a+b e)(1+b(m-1))\}
$$

and let $\tilde{\mu}_{m}^{(1)} \leq \cdots \leq \tilde{\mu}_{m}^{(m)}$ be the eigenvalues of the $m \times m$ power GCD matrix $\left(\left(\tilde{W}_{m}\right)^{\varepsilon}\right)$ defined on the set

$$
\tilde{W}_{m}:=\{1,1+b, \ldots, 1+b(m-1)\} .
$$

Thus for $1 \leq i \leq m, \mu_{m}^{(i)}=(a+b e)^{\varepsilon} \cdot \tilde{\mu}_{m}^{(i)}$. In particular,

$$
\mu_{m}^{(q)}=(a+b e)^{\varepsilon} \tilde{\mu}_{m}^{(q)} .
$$

Now let

$$
m_{n}:=1+\left\lfloor\frac{n-1}{a+b e}\right\rfloor,
$$

where $\lfloor x\rfloor$ denotes the largest integer $\leq x$. Choose $n$ so that $m_{n} \geq q$.

By Cauchy's interlacing inequalities

$$
\lambda_{n}^{(q)} \leq \mu_{m_{n}}^{(q)}
$$

and by (3.9) and (3.10),

$$
\lambda_{n}^{(q)} \leq(a+b e)^{\varepsilon} \tilde{\mu}_{m_{n}}^{(q)}
$$

By Lemma 3.2

$$
\lim _{m \rightarrow \infty} \tilde{\mu}_{m}^{(q)}=0
$$

so

$$
\lim _{n \rightarrow \infty} \tilde{\mu}_{m_{n}}^{(q)}=0
$$

By Proposition 1.1 and (3.11) we get

$$
\lim _{n \rightarrow \infty} \lambda_{n}^{(q)}=0 .
$$

Furthermore, applying again Cauchy's interlacing inequalities, it follows from Proposition 1.1 and Theorem 3.3 that the following result holds. 
THEOREM 3.4. Let $a, b \geq 1$ and $e \geq 0$ be integers and $0<\varepsilon \leq 1$. Let $\left\{x_{i}\right\}_{i=1}^{\infty}$ be any given strictly increasing infinite sequence of positive integers which contains the arithmetic progression $\{a+b i\}_{i=e}^{\infty}$ as its subsequence. Let $\lambda_{n}^{(1)} \leq \cdots \leq \lambda_{n}^{(n)}$ be the eigenvalues of the $n \times n$ power GCD matrix $\left(\left(x_{i}, x_{j}\right)^{\varepsilon}\right)$ defined on the set $S_{n}=\left\{x_{1}, \ldots, x_{n}\right\}$. Then for any given integer $q \geq 1$

$$
\lim _{n \rightarrow \infty} \lambda_{n}^{(q)}=0
$$

Finally we give the following immediate consequence as the conclusion of this section.

COROllary 3.5. Let $0<\varepsilon \leq 1$. Let $\lambda_{n}^{(1)} \leq \cdots \leq \lambda_{n}^{(n)}$ be the eigenvalues of the $n \times n$ power GCD matrix $\left((i, j)^{\varepsilon}\right)$ defined on the set $\bar{S}_{n}=\{1, \ldots, n\}$. Then for any given integer $q \geq 1$

$$
\lim _{n \rightarrow \infty} \lambda_{n}^{(q)}=0
$$

4. Lower bound for the smallest eigenvalue and limit behavior of the $q$-th largest eigenvalue. In this section, we assume always that $1 \leq x_{1}<\cdots<x_{n}<\cdots$ is an arbitrary given infinite sequence of positive integers. Let $\varepsilon>0$ be any given real number. Let $S_{n}=\left\{x_{1}, \ldots, x_{n}\right\}$. Let $\lambda_{n}^{(1)} \leq \cdots \leq \lambda_{n}^{(n)}$ be the eigenvalues of the $n \times n$ power GCD matrix $\left(\left(S_{n}\right)^{\varepsilon}\right)$ defined on the set $S_{n}$. By Bourque and Ligh's result [4] the matrix $\left(\left(S_{n}\right)^{\varepsilon}\right)$ is positive definite and so $\lambda_{n}^{(i)}>0$ for all $1 \leq i \leq n$. We will improve this result by giving a lower bound for the smallest eigenvalue $\lambda_{n}^{(1)}$. We need the following structure theorem.

Lemma 4.1. Let $\varepsilon>0$. Let $\bar{S}=\left\{d_{1}, \ldots, d_{m}\right\}$ be a factor closed set (i.e. $\bar{S}$ contains every positive divisor of $d$ for all $d \in \bar{S})$ containing $S_{n}$ and let $A=\left(a_{i j}\right)_{n \times m}$ be the $n \times m$ matrix defined by:

$$
a_{i j}= \begin{cases}\sqrt{J_{\varepsilon}\left(d_{j}\right)}, & \text { if } d_{j} \mid x_{i} \\ 0, & \text { otherwise, }\end{cases}
$$

where $J_{\varepsilon}:=\xi_{\varepsilon} * \mu$ is the generalized Jordan's totient function and $\xi_{\varepsilon}$ is defined by $\xi_{\varepsilon}(x)=$ $x^{\varepsilon}$ for any $x \in \mathbf{Z}$. Then

$$
\left(\left(S_{n}\right)^{\varepsilon}\right)=A \cdot A^{t}
$$

Proof. It follows immediately from [10, Lemma 2].

REMARK. If $\varepsilon$ is a positive integer, then $J_{\varepsilon}$ becomes the Jordan's totient function (see, for example, [1], [20] or [23]). In particular, $J_{1}$ is just Euler's totient function $\varphi$.

Now let $K_{n}$ be the set of all $n \times n$ lower triangular matrices that satisfy: Every main diagonal entry is 1 , and every off-diagonal entry is 0 or 1 . Obviously $K_{n}$ is a finite set of nonsingular matrices and so the set $L_{n}:=\left\{Y \cdot Y^{t}: Y \in K_{n}\right\}$ is also a finite set of $n \times n$ positive definite matrices. Then we can define a positive constant $c_{n}$ depending only on $n$ as follows.

$$
c_{n}:=\min _{Z \in L_{n}}\left\{\mu_{n}^{(1)}(Z): \mu_{n}^{(1)}(Z) \text { is the smallest eigenvalue of } Z\right\} .
$$


We can now give a lower bound for the smallest eigenvalue in terms of the constant $c_{n}$ defined in (4.1) and the generalized Jordan's totient function.

THEOREM 4.2. Let $\varepsilon>0$ and $\left\{x_{i}\right\}_{i=1}^{\infty}$ be an arbitrary given strictly increasing infinite sequence of positive integers. Let $\lambda_{n}^{(1)}$ be the smallest eigenvalue of the $n \times n$ power GCD matrix $\left(\left(x_{i}, x_{j}\right)^{\varepsilon}\right)$ defined on the set $\left\{x_{1}, \ldots, x_{n}\right\}$. Then

$$
\lambda_{n}^{(1)} \geq c_{n} \cdot \min _{1 \leq i \leq n}\left\{J_{\varepsilon}\left(x_{i}\right)\right\}
$$

Proof. Let $A$ be the $n \times m$ matrix defined in Lemma 4.1. Let $S_{n}=\left\{x_{1}, \ldots, x_{n}\right\}$. Then Lemma 4.1 gives

$$
\left(\left(S_{n}\right)^{\varepsilon}\right)=A \cdot A^{t}
$$

Note that given any $m \times m$ permutation matrix $P$ we have

$$
\left(\left(S_{n}\right)^{\varepsilon}\right)=A \cdot A^{t}=A \cdot P P^{t} \cdot A^{t}=(A P) \cdot(A P)^{t} .
$$

Thus we can permute the columns of $A$. So we can assume without loss of any generality that

$$
d_{l}=x_{l}, l=1, \ldots, n
$$

We now partition $A$ as follows:

$$
A=(\overbrace{B}^{n} \mid \overbrace{C}^{m-n})
$$

Therefore

$$
\begin{aligned}
A \cdot A^{t} & =(B \mid C) \cdot\left(\frac{B^{t}}{C^{t}}\right) \\
& =B \cdot B^{t}+C \cdot C^{t} .
\end{aligned}
$$

We introduce the following notation:

Notation. For real symmetric matrices $G_{1}, G_{2}$ of the same order, we write $G_{1} \succeq$ $G_{2} \Longleftrightarrow G_{1}-G_{2}$ is positive semi-definite.

Therefore it follows from (4.2) and (4.3) that

$$
\left(\left(S_{n}\right)^{\varepsilon}\right) \succeq B \cdot B^{t}
$$

Let $\delta_{n}^{(1)} \leq \cdots \leq \delta_{n}^{(n)}$ be the eigenvalues of $B \cdot B^{t}$. Then it is known (see [15]) that $\lambda_{n}^{(i)} \geq \delta_{n}^{(i)}$ for $1 \leq i \leq n$.

Consider now the $n \times n$ matrix $B=\left(b_{i j}\right)$. We have

$$
b_{i j}= \begin{cases}\sqrt{J_{\varepsilon}\left(x_{j}\right)}, & \text { if } x_{j} \mid x_{i} \\ 0, & \text { otherwise }\end{cases}
$$


In particular, $B$ is lower triangular, and the main diagonal entries are $\sqrt{J_{\varepsilon}\left(x_{i}\right)}, i=$ $1, \ldots, n$. We can factor now

$$
B=\tilde{B} \cdot D,
$$

where $D=\operatorname{diag}\left(\sqrt{J_{\varepsilon}\left(x_{1}\right)}, \ldots, \sqrt{J_{\varepsilon}\left(x_{n}\right)}\right)$ and $\tilde{B}=\left(\tilde{b}_{i j}\right)$ is defined by

$$
\tilde{b}_{i j}= \begin{cases}1, & \text { if } x_{j} \mid x_{i} \\ 0, & \text { otherwise. }\end{cases}
$$

Therefore $\tilde{B} \in K_{n}$. So we have

$$
B \cdot B^{t}=\tilde{B} D \cdot D \tilde{B}^{t}=\tilde{B} \cdot D^{2} \cdot \tilde{B}^{t}
$$

and

$$
G:=\left(B \cdot B^{t}\right)^{-1}=\left(\tilde{B}^{t}\right)^{-1} \cdot D^{-2} \cdot \tilde{B}^{-1} .
$$

We now use the spectral norm which is denoted by $\|\cdot\|$, and is well known to be the largest singular value of the matrix (see [15]). Let $\rho(\cdot)$ denote the spectral radius. Since $G$ is positive definite, we have

$$
\rho(G)=\|G\|=\left\|\left(\tilde{B}^{t}\right)^{-1} \cdot D^{-2} \cdot \tilde{B}^{-1}\right\| \leq \alpha \cdot\left\|\left(\tilde{B}^{t}\right)^{-1}\right\| \cdot\left\|\tilde{B}^{-1}\right\|,
$$

where

$$
\alpha:=\left\|D^{-2}\right\|=\max _{1 \leq i \leq n}\left\{\frac{1}{J_{\varepsilon}\left(x_{i}\right)}\right\}=\frac{1}{\min _{1 \leq i \leq n}\left\{J_{\varepsilon}\left(x_{i}\right)\right\}} .
$$

It is also known that for any matrix $T$,

$$
\left\|T \cdot T^{t}\right\|=\|T\| \cdot\left\|T^{t}\right\|=\|T\|^{2} .
$$

So we have

$$
\rho(G)=\|G\| \leq \alpha \cdot\left\|\left(\left(\tilde{B}^{t}\right)^{-1} \tilde{B}^{-1}\right)\right\|=\alpha \cdot\left\|\left(\tilde{B} \cdot \tilde{B}^{t}\right)^{-1}\right\| .
$$

Since $\tilde{B} \cdot \tilde{B}^{t}$ is positive definite, we have

$$
\left\|\left(\tilde{B} \cdot \tilde{B}^{t}\right)^{-1}\right\|=\rho\left(\left(\tilde{B} \cdot \tilde{B}^{t}\right)^{-1}\right)=\frac{1}{\mu_{n}^{(1)}\left(\tilde{B} \cdot \tilde{B}^{t}\right)},
$$

where $\mu_{n}^{(1)}\left(\tilde{B} \cdot \tilde{B}^{t}\right)$ denotes the smallest eigenvalue of $\tilde{B} \cdot \tilde{B}^{t}$. Therefore it follows from (4.4) that

$$
\rho(G) \leq \frac{\alpha}{\mu_{n}^{(1)}\left(\tilde{B} \cdot \tilde{B}^{t}\right)} \leq \frac{\alpha}{c_{n}}=\frac{1}{c_{n} \cdot \min _{1 \leq i \leq n}\left\{J_{\varepsilon}\left(x_{i}\right)\right\}} .
$$

Since $\rho(G)=\frac{1}{\delta_{n}^{(1)}}$ and $\lambda_{n}^{(1)} \geq \delta_{n}^{(1)}$, we conclude that

$$
\lambda_{n}^{(1)} \geq \frac{1}{\rho(G)} \geq c_{n} \cdot \min _{1 \leq i \leq n}\left\{J_{\varepsilon}\left(x_{i}\right)\right\}
$$

as required. 
COROLlaRY 4.3. Let $\left\{x_{i}\right\}_{i=1}^{\infty}$ be an arbitrary given strictly increasing infinite sequence of positive integers. Let $\lambda_{n}^{(1)}$ be the smallest eigenvalue of the $n \times n \operatorname{GCD}$ matrix $\left(S_{n}\right)$ defined on the set $S_{n}=\left\{x_{1}, \ldots, x_{n}\right\}$. Then

$$
\lambda_{n}^{(1)} \geq c_{n} \cdot \min _{1 \leq i \leq n}\left\{\varphi\left(x_{i}\right)\right\}
$$

Proof. Since $J_{1}(x)=\varphi(x)$ for any positive integer $x$, the result follows immediately from Theorem 4.2.

REMARK. Note that Bourque and Ligh's theorem [4] just states that $\lambda_{n}^{(1)}>0$, so Theorem 4.2 gives a better lower bound for $\lambda_{n}^{(1)}$. Note also that Bourque and Ligh's theorem generalizes a result due to Beslin and Ligh [3] which deals with the special case $\varepsilon=1$.

COROLLARY 4.4. Let $\left\{x_{i}\right\}_{i=1}^{\infty}$ be an arbitrary given strictly increasing infinite sequence of positive integers so that $x_{1}=1$. Let $\lambda_{n}^{(1)}$ be the smallest eigenvalue of the $n \times n$ power GCD matrix $\left(\left(x_{i}, x_{j}\right)^{\varepsilon}\right)$ defined on the set $\left\{x_{1}, \ldots, x_{n}\right\}$. Then for $\varepsilon>0$ we have

$$
\lambda_{n}^{(1)} \geq c_{n} .
$$

Proof. Since $J_{\varepsilon}(1)=1$ and $J_{\varepsilon}(x) \geq 1$ for any integer $x \geq 2$, the result follows immediately from Theorem 4.2.

LEMMA 4.5. Let $x>1$ be a positive integer.

(i) If $\varepsilon>1$, then

$$
J_{\varepsilon}(x) \geq \frac{x^{\varepsilon}}{\zeta(\varepsilon)}
$$

(ii) For $\varepsilon=1$, we have

$$
J_{1}(x) \geq \frac{x \cdot e^{-\gamma}}{\log x}\left(1-\frac{C}{\log x}\right)
$$

where $C>0$ is a constant and $\gamma$ is Euler's constant.

(iii) For $0<\varepsilon<1$, we have

$$
J_{\varepsilon}(x)=x^{\varepsilon(1-\delta)} \cdot g(x),
$$

where $0<\delta<1$ is a constant and $g(x)$ is a function depending only on $\varepsilon$ and $\delta$ satisfying that $g(x) \rightarrow \infty$ as $x \rightarrow \infty$.

(iv) If $\varepsilon>0$, then $\lim _{x \rightarrow \infty} J_{\varepsilon}(x)=\infty$.

Proof. First for any real number $\varepsilon, J_{\varepsilon}$ is multiplicative. For a prime $p$ and a positive integer $l$,

$$
J_{\varepsilon}\left(p^{l}\right)=\left(\xi_{\varepsilon} * \mu\right)\left(p^{l}\right)=p^{l \varepsilon}\left(1-\frac{1}{p^{\varepsilon}}\right)
$$

So we have

$$
J_{\varepsilon}(x)=x^{\varepsilon} \prod_{p \mid x}\left(1-\frac{1}{p^{\varepsilon}}\right) .
$$


It follows immediately that for $\varepsilon>0$

$$
J_{\varepsilon}(x) \geq x^{\varepsilon} \prod_{p \in I}\left(1-\frac{1}{p^{\varepsilon}}\right),
$$

where $I$ denotes the set of all positive prime numbers.

If $\varepsilon>1$, then Euler's formula (see, for example, [1] or [17]) says that

$$
\zeta(\varepsilon)=\prod_{p \in I}\left(1-\frac{1}{p^{\varepsilon}}\right)^{-1}
$$

where $\zeta$ means the usual Riemann's zeta function. So we have

$$
\prod_{p \in I}\left(1-\frac{1}{p^{\varepsilon}}\right)=\frac{1}{\zeta(\varepsilon)}
$$

Therefore (i) follows from (4.6) and (4.7).

If $\varepsilon=1$, by (4.5) we have

$$
J_{1}(x) \geq x \prod_{p \leq x}\left(1-\frac{1}{p}\right) .
$$

But a theorem of Mertens (see [1], or [23], or [24]) gives

$$
\prod_{p \leq x}\left(1-\frac{1}{p}\right)=\frac{e^{-\gamma}}{\log x}\left(1+O\left(\frac{1}{\log x}\right)\right),
$$

where $\gamma$ is Euler's constant. So there exists a constant $C>0$ such that

$$
\prod_{p \leq x}\left(1-\frac{1}{p}\right) \geq \frac{e^{-\gamma}}{\log x}\left(1-\frac{C}{\log x}\right) .
$$

Now (ii) follows immediately from (4.8) and (4.9).

Let now $0<\varepsilon<1$. Write

$$
f(x)=\frac{x^{\varepsilon(1-\delta)}}{J_{\varepsilon}(x)} .
$$

Then $f(x)$ is multiplicative. We claim that $f(x) \rightarrow 0$ when $x \rightarrow \infty$. By Theorem 316 of [7], it is sufficient to prove that $f\left(p^{m}\right) \rightarrow 0$ when $p^{m} \rightarrow \infty$. But

$$
\frac{1}{f\left(p^{m}\right)}=p^{m \varepsilon \delta}\left(1-\frac{1}{p^{\varepsilon}}\right) \rightarrow \infty
$$

as $p^{m} \rightarrow \infty$. Therefore the claim is proved. Now let $g=\frac{1}{f}$. Then $g(x) \rightarrow \infty$ when $x \rightarrow \infty$ and $J_{\varepsilon}(x)=x^{\varepsilon(1-\delta)} \cdot g(x)$ as desired. Thus (iii) is proved.

The statement of (iv) follows immediately from (i)-(iii).

COROLLARY 4.6. Let $\left\{x_{i}\right\}_{i=1}^{\infty}$ be an arbitrary given strictly increasing infinite sequence of positive integers so that $x_{1}>1$. Let $\lambda_{n}^{(1)}$ be the smallest eigenvalue of the $n \times n$ power 
GCD matrix $\left(\left(x_{i}, x_{j}\right)^{\varepsilon}\right)$ defined on the set $\left\{x_{1}, \ldots, x_{n}\right\}$. Then for $\varepsilon>1$ we have

$$
\lambda_{n}^{(1)} \geq c_{n} \cdot \frac{x_{1}^{\varepsilon}}{\zeta(\varepsilon)} .
$$

For $\varepsilon=1$, we have

$$
\lambda_{n}^{(1)} \geq c_{n} \cdot \min _{1 \leq i \leq n}\left\{\frac{x_{i} \cdot e^{-\gamma}}{\log x_{i}}\left(1-\frac{C}{\log x_{i}}\right)\right\},
$$

where $C>0$ is a constant and $\gamma$ is Euler's constant;

For $0<\varepsilon<1$, we have

$$
\lambda_{n}^{(1)} \geq c_{n} \cdot \min _{1 \leq i \leq n}\left\{x_{n-i}^{\varepsilon(1-\delta)} \cdot g\left(x_{n-i}\right)\right\}
$$

where $0<\delta<1$ is a constant and $g(x)$ is the function defined in Lemma 4.5.

Proof. It follows from Theorem 4.2 and Lemma 4.5.

REMARK. If $x$ is sufficiently large, then $\frac{x}{\log x}\left(1-\frac{C}{\log x}\right)$ is strictly increasing we deduce that if $x_{1}$ is sufficiently large, then (4.10) becomes

$$
\lambda_{n}^{(1)} \geq c_{n} \cdot \frac{x_{1} \cdot e^{-\gamma}}{\log x_{1}}\left(1-\frac{C}{\log x_{1}}\right) .
$$

THEOREM 4.7. Let $q \geq 1$ be an arbitrary given integer and $\left\{x_{i}\right\}_{i=1}^{\infty}$ an arbitrary given strictly increasing infinite sequence of positive integers. Let $\lambda_{n}^{(1)} \leq \cdots \leq \lambda_{n}^{(n)}$ be the eigenvalues of the $n \times n$ power GCD matrix $\left(\left(x_{i}, x_{j}\right)^{\varepsilon}\right)$ defined on the set $\left\{x_{1}, \ldots, x_{n}\right\}$. Then, if $n>q$, the following four statements hold.

(i) For $\varepsilon>1$,

$$
\lambda_{n}^{(n-q+1)} \geq c_{q} \cdot \frac{x_{n-q+1}^{\varepsilon}}{\zeta(\varepsilon)}
$$

(ii) For $\varepsilon=1$,

$$
\lambda_{n}^{(n-q+1)} \geq c_{q} \cdot \min _{0 \leq i \leq q-1}\left\{\frac{x_{n-i} \cdot e^{-\gamma}}{\log x_{n-i}}\left(1-\frac{C}{\log x_{n-i}}\right)\right\},
$$

where $C>0$ is a constant and $\gamma$ is Euler's constant.

(iii) For $0<\varepsilon<1$,

$$
\lambda_{n}^{(n-q+1)} \geq c_{q} \cdot \min _{0 \leq i \leq q-1}\left\{x_{i}^{\varepsilon(1-\delta)} \cdot g\left(x_{i}\right)\right\},
$$

where $0<\delta<1$ is a constant and $g(x)$ is the function defined in Lemma 4.5.

(iv) For $\varepsilon>0, \lim _{n \rightarrow \infty} \lambda_{n}^{(n-q+1)}=\infty$.

Proof. Let $R_{q}=\left(\left(x_{n-i}, x_{n-j}\right)^{\varepsilon}\right)$ be the $q \times q$ power GCD matrix defined on the set $\left\{x_{n-q+1}, \ldots, x_{n}\right\}$. Let $\mu_{q}^{(1)} \leq \cdots \leq \mu_{q}^{(q)}$ be the eigenvalues of $R_{q}$. Then Theorem 4.2 applied to $R_{q}$ gives

$$
\mu_{q}^{(1)} \geq c_{q} \cdot \min _{0 \leq i \leq q-1}\left\{J_{\varepsilon}\left(x_{n-i}\right)\right\}
$$


where $c_{q}$ is a constant depending only on $q$ and is defined by (4.1). By Cauchy's interlacing inequalities we have

$$
\lambda_{n}^{(n-q+1)} \geq \mu_{q}^{(1)}
$$

So we have

$$
\lambda_{n}^{(n-q+1)} \geq c_{q} \cdot \min _{0 \leq i \leq q-1}\left\{J_{\varepsilon}\left(x_{n-i}\right)\right\} .
$$

By Lemma 4.5 applied to $J_{\varepsilon}\left(x_{n-i}\right)$ for $0 \leq i \leq q-1$, the statements of (i)-(iii) follow immediately from (4.12).

The statement of (iv) follows immediately from the lower bounds of (i)-(iii).

REMARKS.

1. If $\varepsilon=1$ and $n$ is sufficiently large, then (4.11) becomes

$$
\lambda_{n}^{(n-q+1)} \geq c_{q} \cdot \frac{x_{n-q+1} \cdot e^{-\gamma}}{\log x_{n-q+1}}\left(1-\frac{C}{\log x_{n-q+1}}\right) .
$$

2. Mertens' theorem gives an asymptotic formula for the following product

$$
\prod_{p \leq x}\left(1-\frac{1}{p^{\varepsilon}}\right)
$$

if $\varepsilon=1$. We believe that there exists a similar but more complicated asymptotic formula for the product (4.13) for the case $0<\varepsilon<1$. Such asymptotic formula should give a more explicit lower bound for $J_{\varepsilon}(x)$ and hence for $\lambda_{n}^{(1)}$ and for $\lambda_{n}^{(n-q+1)}$ if $0<\varepsilon<1$.

5. Concluding remarks and questions. Let $\left\{x_{i}\right\}_{i=1}^{\infty}$ be an arbitrary strictly increasing infinite sequence of positive integers. For an integer $n \geq 1$, let $S_{n}=$ $\left\{x_{1}, \ldots, x_{n}\right\}$. Let $0<\varepsilon \leq 1$ and $q \geq 1$ a given integer. Let $\lambda_{n}^{(1)} \leq \cdots \leq \lambda_{n}^{(n)}$ be the eigenvalues of the power GCD matrix $\left(\left(x_{i}, x_{j}\right)^{\varepsilon}\right)$ defined on the set $S_{n}$. It follows from Theorem 2.5 that if for every $i \neq j, \quad\left(x_{i}, x_{j}\right)=x_{1}$ and $\sum_{i=1}^{\infty} \frac{1}{x_{i}}=\infty$, then $\lim _{n \rightarrow \infty} \lambda_{n}^{(1)}=0$. Then by Cauchy's interlacing inequalities and Proposition 1.1 we have that for any given strictly increasing infinite sequence $\left\{x_{i}\right\}_{i=1}^{\infty}$ of positive integers which contains a subsequence $\left\{x_{i}^{\prime}\right\}_{i=1}^{\infty}$ satisfying that for every $i \neq j, \quad\left(x_{i}^{\prime}, x_{j}^{\prime}\right)=x_{1}^{\prime}$ and $\sum_{i=1}^{\infty} \frac{1}{x_{i}^{\prime}}=\infty, \lim _{n \rightarrow \infty} \lambda_{n}^{(1)}=0$. On the other hand, by Theorem 3.4 we know that for any given strictly increasing infinite sequence $\left\{x_{i}\right\}_{i=1}^{\infty}$ of positive integers containing the arithmetic progression $\{a+b i\}_{i=e}^{\infty}$ as its subsequence, $\lim _{n \rightarrow \infty} \lambda_{n}^{(q)}=0$. First we would like to understand for what sequences $\left\{x_{i}\right\}_{i=1}^{\infty}, \lim _{n \rightarrow \infty} \lambda_{n}^{(1)}=0$. Namely, we have the following question:

QUESTION 5.1. Characterize all strictly increasing infinite sequences $\left\{x_{i}\right\}_{i=1}^{\infty}$ of positive integers so that $\lim _{n \rightarrow \infty} \lambda_{n}^{(1)}=0$, where $\lambda_{n}^{(1)}$ is the smallest eigenvalue of the power GCD matrix $\left(\left(x_{i}, x_{j}\right)^{\varepsilon}\right)$ defined on the set $\left\{x_{1}, \ldots, x_{n}\right\}$ and $\varepsilon$ is a positive real number.

Consequently we propose a further problem.

QUESTION 5.2. Given any integer $q \geq 1$, characterize all strictly increasing infinite sequences $\left\{x_{i}\right\}_{i=1}^{\infty}$ of positive integers so that $\lim _{n \rightarrow \infty} \lambda_{n}^{(q)}=0$, where $\lambda_{n}^{(q)}$ is the $q$-th 
smallest eigenvalue of the power GCD matrix $\left(\left(x_{i}, x_{j}\right)^{\varepsilon}\right)$ defined on the set $\left\{x_{1}, \ldots, x_{n}\right\}$ and $\varepsilon$ is a positive real number.

Finally, we suggest a conjecture as the conclusion of this paper.

Conjecture 5.3. Let $\varepsilon>1$ and $\left\{x_{i}\right\}_{i=1}^{\infty}$ be an arbitrary given strictly increasing infinite sequence of positive integers. Let $\lambda_{n}^{(1)}$ be the smallest eigenvalue of the $n \times n$ power GCD matrix $\left(\left(x_{i}, x_{j}\right)^{\varepsilon}\right)$ defined on the set $S_{n}=\left\{x_{1}, \ldots, x_{n}\right\}$. Then $\lim _{n \rightarrow \infty} \lambda_{n}^{(1)}>0$.

AcKnowledgements. The first author was a Lady Davis Fellow at the Technion from July 2002 to July 2003. The first author's research was supported by the Lady Davis Fellowship at the Technion and a grant of NNSF of China (Grant No. 10101015). The second author's research was supported by the Fund for the Promotion of Research at the Technion.

\section{REFERENCES}

1. T. M. Apostol, Introduction to analytic number theory (Springer-Verlag, 1976).

2. T. M. Apostol, Arithmetical properties of generalized Ramanujan sums, Pacific J. Math. 41 (1972), 281-293.

3. S. Beslin and S. Ligh, Greatest common divisor matrices, Linear Algebra Appl. 118 (1989), 69-76.

4. K. Bourque and S. Ligh, Matrices associated with arithmetical functions, Linear and Multilinear Algebra 34 (1993), 261-267.

5. K. Bourque and S. Ligh, Matrices associated with classes of arithmetical functions, J. Number Theory 45 (1993), 367-376.

6. K. Bourque and S. Ligh, Matrices associated with classes of multiplicative functions, Linear Algebra Appl. 216 (1995), 267-275.

7. G. H. Hardy and E. M. Wright, An introduction to the theory of numbers, Fourth Edition (Oxford University Press, 1960).

8. P. Haukkanen, J. Wang and J. Sillanpää, On Smith's determiant, Linear Algebra Appl. 258 (1997), 251-269.

9. H. Hedenmalm, P. Lindqvist and K. Seip, A Hilbert space of Dirichlet series and systems of dilated functions in $L^{2}(0,1)$, Duke Math. J. 86 (1997), 1-37.

10. S. Hong, Bounds for determinants of matrices associated with classes of arithmetical functions, Linear Algebra Appl. 281 (1998), 311-322.

11. S. Hong, On the Bourque-Ligh conjecture of least common multiple matrices, J. Algebra 218 (1999), 216-228.

12. S. Hong, GCD-closed sets and determinants of matrices associated with arithmetical functions, Acta Arith. 101 (2002), 321-332.

13. S. Hong, Factorization of matrices associated with classes of arithmetical functions, Colloquium Math. 98 (2003), 113-123.

14. S. Hong, Notes on power LCM matrices, Acta Arith. 111 (2004), 165-177.

15. R. Horn and C. R. Johnson, Matrix analysis (Cambridge University Press, 1985). 1991).

16. R. Horn and C. R. Johnson, Topics in matrix analysis (Cambridge University Press,

17. K. Ireland and M. Rosen, A classical introduction to modern number theory, Second Edition (Springer-Verlag, 1990).

18. I. Korkee and P. Haukkanen, On meet and join matrices associated with incidence function, Linear Algebra Appl. 372 (2003), 127-153.

19. P. Lindqvist and K. Seip, Note on some greatest common divisor matrices, Acta Arith. 84 (1998), 149-154.

20. P. J. McCarthy, Introduction to arithmetical functions (Springer-Verlag, New York, 1986).

21. P. J. McCarthy, A generalization of Smith's determinant, Canad. Math. Bull. 29 (1988), $109-113$. 
22. F. Mertens, Ein Beitrag zur analytischen Zahlentheorie, J. Reine Angew. Math. 78(1874), $46-62$.

23. M. R. Murty, Problems in analytic number theory (Springer-Verlag, 2001).

24. W. Narkiewicz, The development of prime number theory, from Euclid to Hardy and Littlewood (Springer-Verlag, 2000).

25. H. J. S. Smith, On the value of a certain arithmetical determinant, Proc. London Math. Soc. 7 (1875-1876), 208-212.

26. A. Wintner, Diophantine approximations and Hilbert's space, Amer. J. Math. 66 (1944), 564-578. 